\title{
Les boiteries dans un atelier de taurillons à l'engrais : observations cliniques et lésionnelles, relations avec la croissance
}

\author{
F. SERIEYS, P.L. GASTINEL et Y.M. CHATELIN \\ Institut Technique de l'Elevage bovin \\ 149, rue de Bercy, F 75595 Paris Cedex 12
}

\begin{abstract}
Résumé
Des observations systématiques des boiteries ont été réalisées pendant quatorze mois sur les taurillons d'une station de testage soumis à un contrôle de croissance mensuel. La fréquence des boiteries a été très élevée puisqu'environ les $2 / 3$ des animaux ont boité, dont 20 p. 100 de façon sévère. Les observations cliniques et les photographies des lésions podales ont révélé que la pododermatite aseptique diffuse était à l'origine de la plupart des boiteries. L'examen des conditions d'alimentation pratiquées dans cet élevage n'a pas permis d'attribuer aux facteurs nutritionnels et métaboliques un rôle étiologique prédominant par rapport aux facteurs traumatiques liés aux conditions de logement des animaux sur sol dur sans paille, à double pente à 8 p. 100 avec caillebotis central. Le rôle possible de chacun de ces facteurs et de leur combinaison a été discuté.

L'étude, par analyse discriminante et régression multiple, des relations entre boiteries et vitesse de croissance a montré que l'effet propre des boiteries sur le G.M.Q. total était très hautement significatif et se traduisait en cas de boiterie sévère par une diminution du croît de plus de 130 grammes par jour d'engraissement; que les animaux qui boitaient avaient une croissance initiale avant boiterie supérieure à celle des animaux non boiteux. Les implications de ces résultats en matière d'étiologie de la pododermatite aseptique diffuse, d'économie de l'élevage, et de précision des méthodes de sélection, ont été discutées.
\end{abstract}

Mots clés : Boiteries, taurillon, croissance, pododermatite aseptique diffuse.

\section{Introduction}

Les boiteries constituent une pathologie importante chez les taurillons. On estime en effet que les différentes affections de l'appareil locomoteur, par les éliminations et la mortalité qu'elles entraînent, se placent au deuxième rang des problèmes sanitaires rencontrés dans ce type de production, immédiatement après les affections respiratoires (LIBERSA, 1981). Il faudrait ajouter à ces pertes les frais d'intervention vétérinaires, le surcroît de travail et surtout la diminution probable de la vitesse de croissance des animaux boiteux dont l'importance n'a pas encore été évaluée de manière précise. 


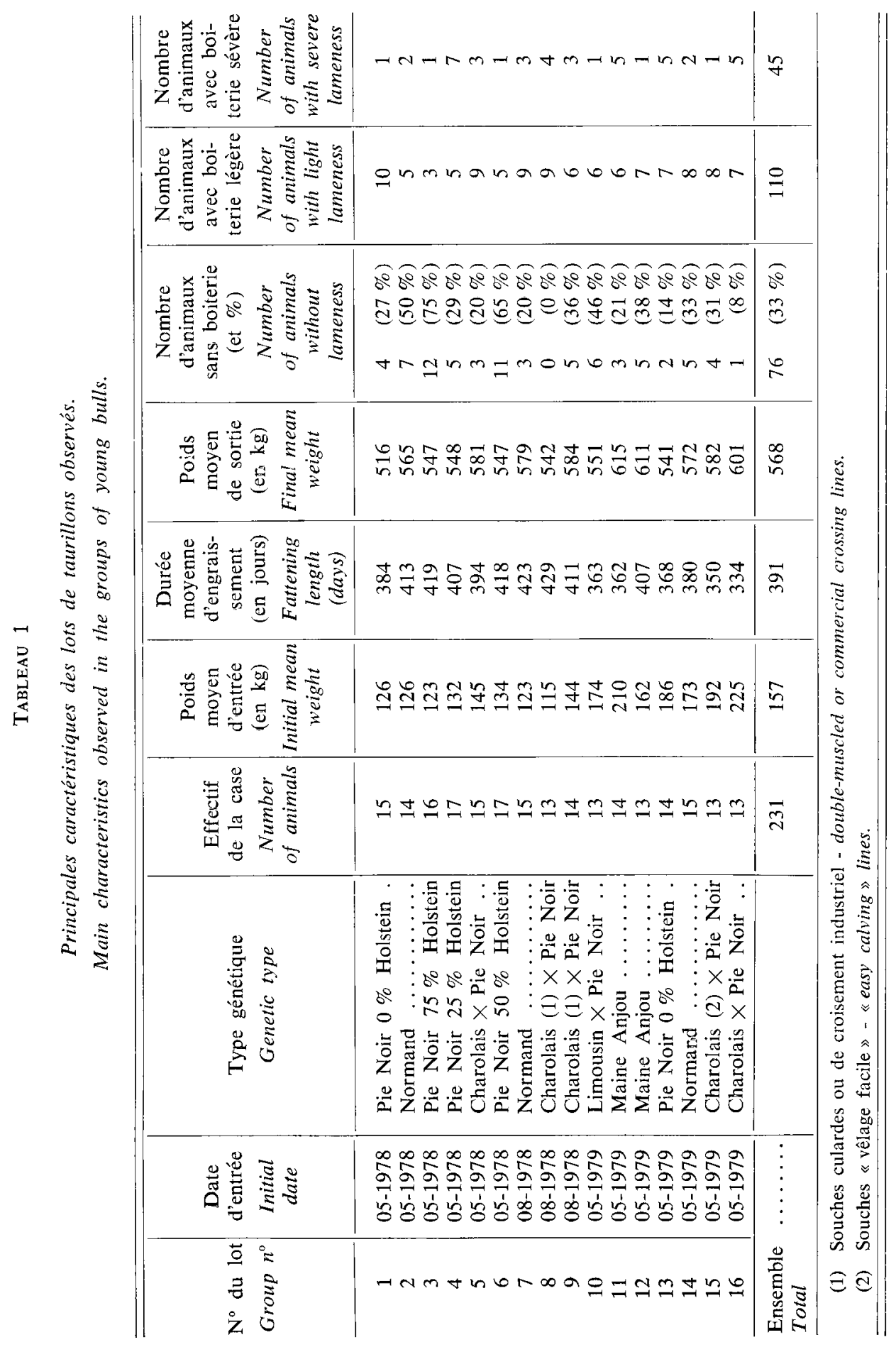


On admet généralement que l'origine des boiteries est multiple et fait intervenir, outre des facteurs génétiques, physiologiques et infectieux, les conditions de logement et d'alimentation (Bezille, Boccara \& Paragon, 1978).

Plus particulièrement la fourbure, dont l'apparition est généralement associée à des conditions d'élevage intensif que ce soit en production de lait ou de viande, est considérée comme une maladie d'étiologie complexe où dominent les facteurs alimentaires et métaboliques (GreENOUGH, 1982).

C'est une cause fréquente de boiteries chez les jeunes bovins à l'engrais et il est possible dans ce type d'élevage, en récupérant les pieds à l'abattoir dans un délai assez bref après les manifestations de boiteries, de mettre en évidence les lésions podales caractéristiques de la maladie. En outre, le mode de conduite en atelier spécialisé, avec des lots d'animaux assez homogènes au plan physiologique, placés dans des conditions d'environnement comparables et recevant des régimes alimentaires simples, peut faciliter la mise en évidence de certains facteurs étiologiques.

Dans ce but et afin de préciser les relations entre les boiteries et la vitesse de croissance, une observation systématique a été réalisée pendant quatorze mois dans un atelier de taurillons.

\section{Matériel et méthodes}

L'observation a été réalisée entre février 1979 et avril 1980, à la station de testage pour la production de jeunes bovins de Saint-Lubin (Loir-et-Cher), appartenant à l'Union Régionale Centre-Ouest des Coopératives d'insémination artificielle. Elle a concerné 231 taurillons de 8 types génétiques différents, entrés en trois groupes sur une période d'un an. On a ainsi constitué 16 lots dont les principales caractéristiques sont résumées dans le tableau 1. Pendant la période d'engraissement, les animaux recevaient à volonté de l'ensilage de maïs à forte teneur en matière sèche (entre 30 et 38 p. 100 de M.S. selon les silos) complété en azote, minéraux, oligo-éléments et vitamines. En outre des blocs à lécher contenant des oligo-éléments étaient en permanence à leur disposition. Les animaux étaient logés dans un bâtiment sans paille, du type double pente à 8 p. 100 avec caillebotis central (fig. 1), équipé d'un réseau électrique basse tension à 1,60 mètre de hauteur pour limiter les chevauchements.

La surface disponible par animal, pour 15 animaux par case, était de $2 \mathrm{~m}^{2}$.

Les animaux étaient observés quotidiennement au moment de la distribution d'ensilage. Dès lors qu'un animal manifestait des difficultés de déplacement, il était considéré comme boiteux, même en l'absence de claudication marquée. Pour chaque boiterie observée on a enregistré la date d'apparition, la durée, la gravité selon une échelle de 1 à 4, la localisation, les anomalies d'onglons, les autres signes cliniques, le traitement appliqué. Par ailleurs, pour une partie des animaux boiteux, les pieds ont été récupérés à l'abattoir pour une recherche systématique des lésions podales. Enfin la croissance des animaux a été suivie par des pesées mensuelles.

Selon leur situation vis-à-vis des boiteries, on a distingué trois groupes d'animaux : ceux qui n'ont jamais boité au cours de la période d'engraissement (B1), ceux ayant manifesté une boiterie légère n'ayant pas nécessité l'isolement à l'infirmerie (B2) et enfin ceux qui ont été atteints de boiterie sévère avec passage à l'infirmerie (B3). 


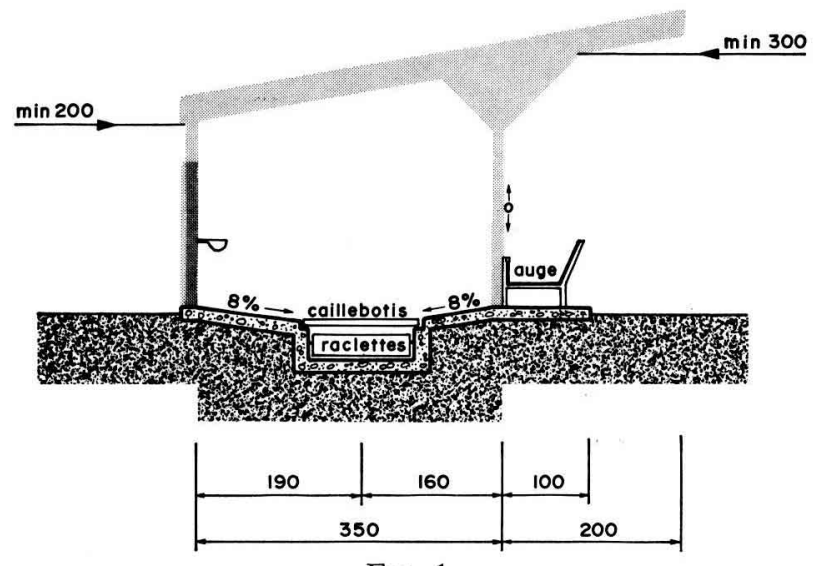

FIG. 1

Plan schématique du bâtiment.

House plan.

Une analyse discriminante de ces groupes d'animaux a été effectuce en fonction :

- du poids à l'entrée en engraissement $(\mathbf{P o})$;

- du gain moyen quotidien initial sur la période allant de la date d'entrée jusqu'à la date de $1^{\text {re }}$ boiterie du lot (GI);

- du gain moyen quotidien final sur la période allant de la date de $1^{\text {re }}$ boiterie du lot jusqu'à la date de sortie (GF); (GT).

- du gain moyen quotidien total sur l'ensemble de la période d'engraissement

Dans cette analyse, n'ont pu être considérés que les animaux ayant commencé à boiter plus de 4 mois après leur entrée, ce qui a conduit à éliminer 13 animaux ayant boité plus précocément. Dans ces conditions, la durée de la période entrée-première boiterie du lot a été comprise, selon les lots, entre 120 et 126 jours.

Une analyse de variance-covariance a également été effectuée sur le modèle suivant :

$$
\mathrm{GT}_{\mathrm{ijk}}=\mathrm{C}+\mathrm{B}_{\mathrm{i}}+\mathbf{L}_{\mathbf{j}}+\alpha \mathrm{Po}_{\mathrm{ijk}}+\beta \mathrm{GI}_{\mathrm{ijk}}+\varepsilon_{\mathrm{ijk}}
$$

avec $\mathrm{GT}_{\mathrm{ijk}}$ : gain moyen quotidien de l'animal $\mathrm{k}$ du lot $\mathrm{j}$, dans la situation i vis-àvis 'des boiteries

C : terme constant

$B_{i} \quad$ : effet commun aux animaux dans une même situation vis-à-vis des boiteries avec $\mathrm{i}=1,2,3$

$\mathrm{L}_{\mathrm{j}} \quad$ : effet commun aux animaux d'un même lot (j variant de 1 à 16)

$\alpha \quad$ : coefficient de régression de la variable poids à l'entrée en engraissement (Po)

$\beta \quad$ : coefficient de régression de la variable gain moyen quotidien initial (GI)

$\varepsilon_{\mathrm{ijk}} \quad:$ résidu aléatoire 
Il s'agit d'un modèle déséquilibré (proportions d'animaux boiteux variables selon les lots), et l'estimation des effets a été obtenue par calcul matriciel.

\section{III. - Résultats}

\section{A. Fréquence des boiteries}

Le tableau 1 fait apparaître la forte fréquence des boiteries dans cet élevage puisque, sur 231 animaux observés, 76 seulement, soit un tiers environ, n’ont présenté aucun signe alors que 45 animaux, soit plus d'un animal sur cinq, ont été atteints de boiteries sévères ayant nécessité l'isolement. D'un lot à l'autre les fréquences sont très différentes avec des proportions d'animaux non boiteux variant entre 0 et 75 p. 100 , mais il n'a pas été possible d'imputer de manière précise ces différences à l'un ou l'autre des principaux paramètres qui caractérisent les lots : type génétique, effectif par case, poids à l'entrée, durée d'engraissement, poids à la sortie, qualité de l'ensilage de maïs distribué. On a pu noter toutefois que les périodes d'agitation étaient souvent suivies de plusieurs cas de boiteries et que, d'une case à l'autre, les comportements étaient très différents : pratiquement la présence d'un seul animal chahuteur ou particulièrement excité dans une case entraîne la multiplication des bousculades et semble suffire à augmenter sensiblement la fréquence des boiteries observées dans le lot.

\section{B. Observations cliniques et lésionnelles}

Les premières boiteries sont apparues 9 fois sur 10 au-delà de $300 \mathrm{~kg}$ de poids vif et 3 fois sur 4 au-delà de $350 \mathrm{~kg}$ (tabl. 2). Les membres postérieurs ont été concernés plus de 7 fois sur 10 par des boiteries qui dans 95 p. 100 des cas étaient d'origine podale (tabl. 3), les autres cas correspondant surtout à des atteintes articulaires du jarret (arthrite, abcès, jarret droit).

\section{Tableau 2}

Poids d'apparition des boiteries (lots d'animaux entrés en août 1978 et mai 1979).

Weight ranges and occurrence of lameness (groups of animals arrived in August 1978 and May 1979).

\begin{tabular}{|c|c|c|}
\hline $\begin{array}{c}\text { Tranche de poids } \\
\text { Weight range }\end{array}$ & $\begin{array}{l}\text { Nombre d'animaux boiteux } \\
\text { Number of lameness cases }\end{array}$ & $\%$ \\
\hline$-250 \mathrm{~kg}$ & 1 & 1 \\
\hline 250 à $300 \mathrm{~kg} \ldots \ldots \ldots \ldots \ldots \ldots$ & 9 & 8,7 \\
\hline 300 à $350 \mathrm{~kg} \ldots \ldots \ldots \ldots \ldots \ldots$ & 18 & 17,5 \\
\hline 350 à $400 \mathrm{~kg} \ldots$ & 26 & 25,2 \\
\hline 400 à $450 \mathrm{~kg} \quad \ldots$ & 25 & 24,3 \\
\hline$+450 \mathrm{~kg} \ldots \ldots \ldots \ldots \ldots$ & 24 & 23,3 \\
\hline Ensemble - Total & 103 & $100 \%$ \\
\hline
\end{tabular}




\section{TABleau 3}

Localisation des boiteries.

Localization of lamenesses.

\begin{tabular}{|c|c|c|}
\hline $\begin{array}{l}\text { Localisation } \\
\text { Localization }\end{array}$ & $\begin{array}{l}\text { Nombre de boiteries } \\
\text { Number of lamenesses }\end{array}$ & $\%$ \\
\hline $\begin{array}{l}\text { Postéricur droit } \ldots \ldots \ldots \ldots \ldots \ldots \\
\text { Right hindly }\end{array}$ & 79 & 35 \\
\hline $\begin{array}{l}\text { Postérieur gauche } \ldots \ldots \ldots \ldots \ldots \\
\text { Right hindly }\end{array}$ & 85 & 38 \\
\hline $\begin{array}{l}\text { Antérieur droit } \ldots \ldots \ldots \ldots \ldots \ldots \\
\text { Right forely }\end{array}$ & 34 & 15 \\
\hline $\begin{array}{l}\text { Antérieur gauche } \ldots \ldots \ldots \ldots \\
\text { Left forely }\end{array}$ & 27 & 12 \\
\hline Ersemble - Total .. & 225 & $100 \%$ \\
\hline $\begin{array}{l}\text { Localisation podale } \ldots \ldots \ldots \ldots \ldots \\
\text { Podal localization }\end{array}$ & 214 & 95 \\
\hline $\begin{array}{l}\text { Au-dessus du pied } \ldots \ldots \ldots \\
\text { Above the foot }\end{array}$ & 11 & 5 \\
\hline Ersemble - Total ........... & 225 & $100 \%$ \\
\hline
\end{tabular}

\section{TABLeAU 4}

Répartition des affections podales identifiées.

Distribution of identified podal lesions.

\begin{tabular}{|c|c|c|}
\hline $\begin{array}{l}\text { Affection podale identifiée } \\
\text { Identified podal lesion }\end{array}$ & $\begin{array}{l}\text { Nombre } \\
\text { Number }\end{array}$ & $\%$ \\
\hline Panaris interdigité - Foul in the foot ... & 10 & 14 \\
\hline $\begin{array}{l}\text { Pododermatite diffuse sans complications infectieuses } \\
\text { Diffuse pododermatitis } \\
\text { without infectious complications }\end{array}$ & 48 & 69 \\
\hline $\begin{array}{l}\text { Pododermatite diffuse avec complications infectieuses } \\
\text { Diffuse pododermatitis with infectious complications }\end{array}$ & 12 & 17 \\
\hline Ensemble - Total & 70 & $100 \%$ \\
\hline
\end{tabular}

En ce qui concerne les affections podales on a constaté (tabl. 4) : 
- l'incidence ponctuelle de quelques cas de panaris interdigités, répondant très bien au traitement par les sulfamides, et à allure épidémique. Ainsi sur les 10 cas qui ont été observés, 8 sont intervenus dans le lot $n^{\prime \prime} 13$, en moins de 15 jours ;

- l'importance des signes de fourbure ou plus exactement de pododermatite aseptique diffuse (Mahin, Chaddli, Addi, 1982 ; Mortensen \& Hesselholt, 1982) pour s'en tenir aux seules observations cliniques sans préjuger d'une éventuelle étiologie métabolique.

Ainsi 10 animaux ont manifesté très nettement et à différentes reprises at cours de la période d'engraissement des attitudes caractéristiques telles que piétinements permanents, membres ramenés sous le corps, dos voussé, appui sur la pointe des onglons ou sur les talons, positions campées, marche difficile avec raideurs des membres.

Les anomalies d'onglons les plus fréquemment observées au cours de la période d'engraissement ont concerné l'épaississement et l'allongement des onglons avec une sole concave, une production excessive et irrégulière de corne, notamment en sole avec débordement de l'onglon externe sous l'onglon interne, l'usure des talons, des décollements importants en sole, en talon et au niveau de la couronne entraînant un délabrement plus ou moins étendu de l'étui corné.

En outre, on a retrouvé à plusieurs reprises dans le lisier des morceaux de corne d'onglons d'une taille suffisante $\left(10 \mathrm{~cm}^{2}\right.$ environ) pour bloquer le fonctionnement de la pompe.

Au parage, la corne s'est révélée fréquemment hémorragique et de consistance plâtreuse. Enfin, dans les cas graves, l'effraction de l'étui corné était suivie de complications infectieuses avec formation d'abcès internes et nécrose des tissus profonds, d'abcès au niveau du talon, d'inflammations plus ou moins diffuses de la couronne.

Les observations qui ont été réalisées sur 32 pieds d'animaux boiteux récupérés après abattage sont très homogènes et confirment les précédentes avec notamment (planche 1) :

- des décollements étendus de la corne particulièrement au niveau de la sole, avec formation sous-jacente d'une nouvelle corne complètement séparée (photos 1 et 2) ;

- des délabrements profonds de l'onglon notamment en sole et en muraille avec effraction au niveau de la ligne blanche du côté abaxial, aboutissant fréquemment à l'infection des tissus sous-jacents (photos 3,4).

\section{Relations avec la croissance des animaux}

La figure 2 donne une représentation graphique des principaux résultats de l'analyse discriminante. La signification des axes factoriels 1 et 2 est donnée par leurs corrélations avec les variables (tabl. 5). L’axe $\mathrm{n}^{\circ} 1$ qui, avec une part dinertie de 88 p. 100 , est le plus discriminant, représente essentiellement le G.M.Q. final (GF) et dans une moindre mesure le G.M.Q. total. L'axe $n " 2$, nettement moins discriminant avec 12 p. 100 d'inertie, est surtout lié au G.M.Q. initial et dans une moindre mesure au G.M.Q. total (GT) et au poids initial (Po). La position sur le graphique du centre des groupes d'animaux classés selon leur situation vis-à-vis des boiteries (B1, B2, B3) permet de tirer les conclusions suivantes : 


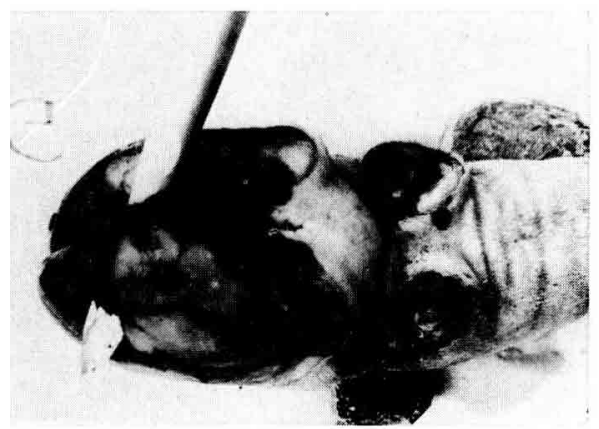

Photo 1

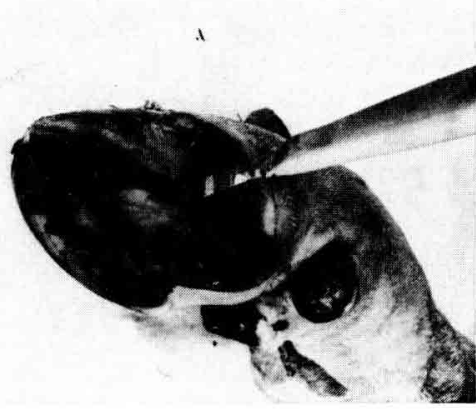

Photo 3

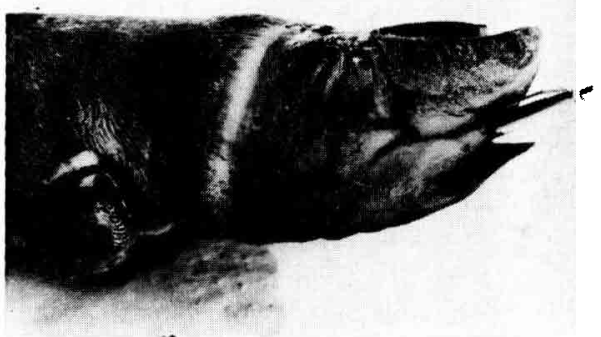

Photo 2

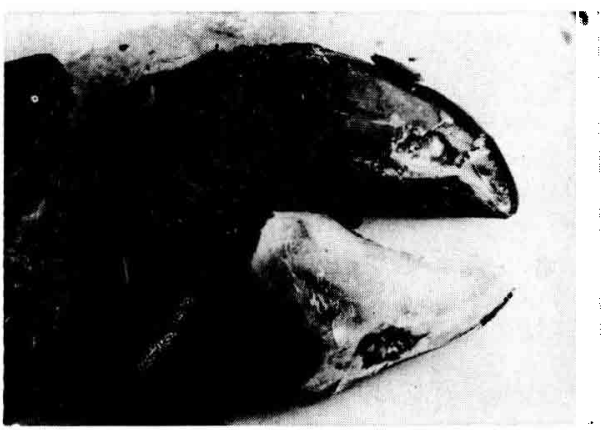

Photo 4

Planche 1

Observations des onglons d'animaux boiteux sur des pieds récupérés après abattage. Post-slaughter observations on hooves of lame animals.

Photos 1 et 2 : Décollement important en sole - Extensive under-running of the sole.

Photos 3 et 4 : Délabrement de l'onglon avec effraction profonde côté abaxial - Disintegration of the hoof with deep penetration of the abaxial side.

\section{TABLEAU 5}

Coefficients de corrélation des variables avec les axes factoriels.

Coefficients of correlation of the variables with the factorial axes.

\begin{tabular}{l|r|r|r|r}
\hline & PO & GI & GF & GT \\
\hline Axe $1 \ldots \ldots \ldots \ldots \ldots$ & $-0,03$ & $-0,23$ & 0,98 & 0,77 \\
Axe $2 \ldots \ldots \ldots \ldots \ldots$ & 0,59 & 0,94 & 0,17 & 0,63 \\
\hline
\end{tabular}

- le G.M.Q. final (GF) des animaux est fortement influencé par leur situation vis-à-vis des boiteries : à partir du moment où les animaux boitent, la vitesse de croissance est ralentie et ceci d'autant plus que les boiteries sont sévères; 
- le groupe d'animaux ayant boité sévèrement (B3) se distingue des deux autres (B1 et B2) par un G.M.Q. total (GT) sur l'ensemble de la période d'engraissement plus faible ;

- les deux groupes d'animaux ayant boité (B2 et B3) se distinguent des animaux n’ayant jamais boité (B1) par une croissance initiale avant boiterie (GI) plus rapide.

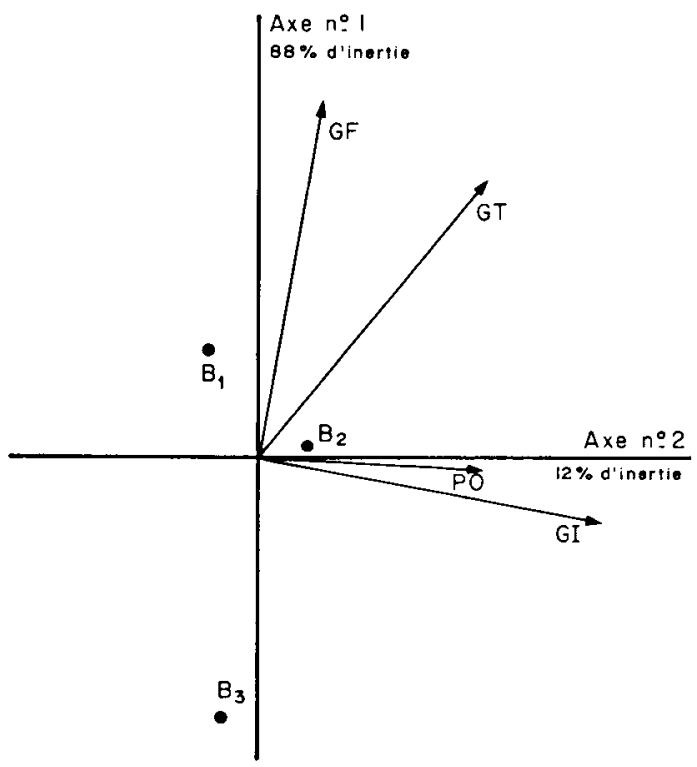

Fig. 2

Graphique d'analyse discriminante.

Discriminatory analysis.

Tableau 6

Corrélations intra-classe (au-dessus de la diagonale) et inter-classes (en-dessous de la diagonale) entre les variables.

Intra-class correlations (above the diagonal) and inter-class correlations (below the diagonal) between variables.

\begin{tabular}{|c|c|c|c|c|}
\hline & PO & GI & GF & GT \\
\hline Po $\ldots \ldots \ldots \ldots \ldots$ & $1-$ & $0,60 * *$ & $-0,03$ & $0,28 * *$ \\
\hline GI $\ldots \ldots \ldots$ & $0,90 * *$ & -1 & $-0,04$ & $0,47 * *$ \\
\hline GF & 0,05 & $-0,49 * *$ & -1 & $0,86 * *$ \\
\hline GT ... & $0,18 *$ & $-0,27 * *$ & $0,97 * *$ & -1 \\
\hline
\end{tabular}

NS : non significatif - not significant.

* significatif au scuil 0,05 - significant at the 0.05 level.

** significatif au seuil 0,01 - significant at the 0.01 level. 
Le tableau des corrélations intra-classes et inter-classes (tabl. 6) permet de préciser l'interprétation. Si l'on considère que le G.M.Q. initial avant boiterie (GI) est un indicateur du potentiel de croissance des animaux, comme le suggèrent les corrélations intra-classes entre les variables GI et GT d'une part, GI et Po d'autre part, il apparaît que les animaux boiteux ont en moyenne un potentiel de croissance supérieur mais qui, du fait de la boiterie, ne peut s'exprimer.

\section{TABLEAU 7}

Effectifs des différents groupes d'animaux et moyennes brutes des variables.

Number of animals in the different groups and gross means of the variables.

\begin{tabular}{l|c|c|c|c|c}
\hline \multicolumn{1}{c|}{$\begin{array}{c}\text { Groupes } \\
\text { Groups }\end{array}$} & $\begin{array}{c}\text { Effectifs } \\
\text { Number } \\
\text { of animals }\end{array}$ & $\begin{array}{c}\text { PO } \\
\text { (Poids entré } \\
\text { en kg) } \\
\text { Initial weight }\end{array}$ & $\begin{array}{c}\text { GI } \\
\text { (G.M.Q. } \\
\text { initial en g) } \\
\text { Initial daily } \\
\text { mean gain }\end{array}$ & $\begin{array}{c}\text { GF } \\
\text { (G.M.Q. } \\
\text { firal en g) } \\
\text { Final daily } \\
\text { mean gain }\end{array}$ & $\begin{array}{c}\text { GT } \\
\text { (G.M.Q. } \\
\text { total en g) } \\
\text { Total daily } \\
\text { mean gain }\end{array}$ \\
\hline $\begin{array}{l}\text { B 1 (non boiteux) ... } \\
\text { No lameness }\end{array}$ & 75 & 148,2 & 1042 & 1086 & 1073 \\
$\begin{array}{l}\text { B 2 (boiterie légère) } \\
\text { Light lameness }\end{array}$ & 105 & 154,2 & 1103 & 1052 & 1069 \\
$\begin{array}{l}\text { B 3 (boiterie sévère) } \\
\text { Severe lameness }\end{array}$ & 36 & 150,1 & 1095 & 924 & 975 \\
\hline $\begin{array}{l}\text { Ensemble ......... } \\
\text { Total }\end{array}$ & 217 & 151 & 1081 & 1042 & 1055 \\
\hline
\end{tabular}

Aussi, l'estimation de la diminution de vitesse de croissance que l'on fait en comparant directement le G.M.Q. total (GT) des animaux ayant boité à celui des animaux n'ayant jamais boité (tabl. 7), est-elle sous évaluée. Une meilleure estimation peut être obtenue par l'étude de la régression du G.M.Q. total (GT) en fonction de la situation vis-à-vis des boiteries (B), du lot (L), du poids d'entrée (Po) et du G.M.Q. initial (GI) : le coefficient de corrélation multiple de cette régression est de 0,63 , les coefficients de l'équation sont donnés dans le tableau 8 et les résultats de l'analyse de variance-covariance dans le tableau 9. Il apparaît alors que l'effet propre des boiteries sur le G.M.Q. total (GT) est très hautement significatif et se traduit par une réduction de vitesse de croissance de $135 \mathrm{~g}$ par jour d'engraissement chez les animaux atteints de boiteries sévères par rapport aux animaux non boiteux. Les moyennes ajustées du G.M.Q. total (GT) sont alors de $1092 \mathrm{~g}$ pour les animaux n'ayant jamais boité, de $1059 \mathrm{~g}$ pour les animaux ayant boité légèrement et de $957 \mathrm{~g}$ pour les animaux ayant boité sévèrement. 


\section{Tableau 8}

Valeur et écart type des coefficients de la régression (variable expliquée : G.M.Q. total).

Value and standard deviation of the coefficients of the regression (explained variable : total daily mean gain).

\begin{tabular}{|c|c|c|c|}
\hline $\begin{array}{l}\text { Variable explicative } \\
\text { Explanatory variable }\end{array}$ & Symbole & $\begin{array}{c}\text { Valeur } \\
\text { des coefficients } \\
\text { Value } \\
\text { of the coefficients }\end{array}$ & $\begin{array}{c}\text { Ecart type } \\
\text { des coefficients } \\
\text { Standard deviation } \\
\text { of the coefficients }\end{array}$ \\
\hline $\begin{array}{l}\text { Terme constant } \\
\text { Constant term }\end{array}$ & $\mathrm{C}$ & 685,50 & \\
\hline $\begin{array}{l}\text { Situation vis-à-vis } \\
\text { des boiteries } \\
\text { Situation relative } \\
\text { to lameness }\end{array}$ & $\begin{array}{l}\mathrm{B} 1 \\
\mathrm{~B} 2 \\
\mathrm{~B} 3\end{array}$ & $\begin{array}{r}56,36 \\
22,80 \\
-79,15\end{array}$ & $\begin{array}{r}9,32 \\
13,22\end{array}$ \\
\hline Lot & $\begin{array}{l}\text { L1 } \\
\text { L2 } \\
\text { L3 } \\
\text { L4 } \\
\text { L5 } \\
\text { L6 } \\
\text { L7 } \\
\text { L8 } \\
\text { L9 } \\
\text { L10 } \\
\text { L11 } \\
\text { L12 } \\
\text { L13 } \\
\text { L14 } \\
\text { L15 } \\
\text { L16 }\end{array}$ & $\begin{array}{r}-11,30 \\
40,99 \\
-19,95 \\
41,44 \\
66,66 \\
-45,70 \\
37,29 \\
28,97 \\
22,70 \\
-46,57 \\
-\quad 0,43 \\
21,49 \\
-79,25 \\
-99,08 \\
19,31 \\
23,41\end{array}$ & $\begin{array}{l}29,01 \\
29,47 \\
29,51 \\
26,58 \\
26,20 \\
27,52 \\
30,10 \\
32,88 \\
27,36 \\
31,26 \\
36,71 \\
27,66 \\
32,10 \\
28,48 \\
33,46\end{array}$ \\
\hline $\begin{array}{l}\text { Poids d'entrée } \\
\text { Initial weight }\end{array}$ & $\alpha$ & 0,39 & 0,48 \\
\hline $\begin{array}{c}\text { G.M.Q. initial } \\
\text { Initial daily mean gain }\end{array}$ & $\beta$ & 0,27 & 0,05 \\
\hline
\end{tabular}




\section{TABleau 9}

Analyse de variance-covariance.

Variance-covariance analysis.

\begin{tabular}{|c|c|c|c|c|}
\hline & $\begin{array}{c}\text { Somme } \\
\text { des carrés } \\
\text { des écarts } \\
\text { Sum of deviation } \\
\text { squares }\end{array}$ & $\begin{array}{c}\text { Degré } \\
\text { de liberté } \\
\text { Degree } \\
\text { of freedom }\end{array}$ & $\begin{array}{l}\text { Carré moyen } \\
\text { Mean square }\end{array}$ & $\mathrm{F}$ \\
\hline $\begin{array}{l}\text { Boiterie - Lameness } \ldots \\
\text { Lot }- \text { Group } \ldots \ldots \ldots \\
\text { PO } \ldots \ldots \ldots \ldots \ldots \ldots \\
\text { GI } \quad \ldots \ldots \ldots \ldots \ldots \ldots\end{array}$ & $\begin{array}{r}388612,61 \\
399773,45 \\
6916,12 \\
316095,52 \\
\end{array}$ & $\begin{array}{r}2 \\
15 \\
1 \\
1\end{array}$ & $\begin{array}{r}194306,30 \\
26651,56 \\
6916,12 \\
316095,52 \\
\end{array}$ & $\begin{array}{r}18,57 * * * \\
2,55 * * \\
0,66 \mathrm{NS} \\
30,21 * * *\end{array}$ \\
\hline Résiduelle - Residual & 2060949,13 & 197 & 10461,67 & \\
\hline Totale & 3421495,68 & 216 & 15840,26 & \\
\hline \multicolumn{5}{|c|}{$\begin{aligned} * * * * & \text { significatif au seuil de } 0,001-P<0.001 \\
* * * & \text { significatif au seuil de } 0,01-P<0.01 \\
\text { NS }: & \text { non significatif }- \text { not significant. }\end{aligned}$} \\
\hline
\end{tabular}

\section{Discussion et conclusions}

Les boiteries sont nombreuses dans l'élevage considéré et entraînent une forte réduction de la vitesse de croissance des animaux. Ainsi peut-on estimer que pour une durée d'engraissement moyenne de 391 jours, la réduction de poids vif due aux boiteries a été d'environ $53 \mathrm{~kg}$ pour les animaux atteints de boiteries sévères et de $13 \mathrm{~kg}$ pour les animaux ayant boité légèrement.

Les observations cliniques et lésionnelles indiquent que la pododermatite aseptique diffuse, avec éventuellement des complications infectieuses secondaires, est à l'origine de la plupart des boiteries observées dans cet élevage, les autres affections comme les arthrites ou les panaris interdigités n'ayant qu'une incidence marginale.

Ces observations conduisent à envisager une étiologie dominée par les facteurs alimentaires et métaboliques. Pourtant l'examen des rations ne révèle aucun déséquilibre en matière azotée, minéraux, oligo-éléments et vitamines et le régime à base d'ensilage de maïs complémenté par 1 à $3 \mathrm{~kg}$ d'un concentré ne présente pas les caractères de concentration énergétique, de fermentescibilité des glucides, de manque de fibres cellulosiques ou de taille réduite des particules, que l'on considère habituellement comme favorisant l'acidose et la fourbure (BRENT, 1976 ; CotTEREaU, 1967 ; Nilsson, 1982). En outre, de nombreux ateliers de taurillons utilisent des régimes analogues sans connaître de problèmes particuliers de boiteries.

Les conditions d'alimentation pratiquées dans cet élevage peuvent donc difficilement expliquer, à elles seules, la fréquence des cas de pododermatite aseptique diffuse observés, et il est très probable que les conditions de logement des animaux joutent 
un rôle important dans l'apparition de ces boiteries. On sait en effet que les sols durs, en favorisant les contusions légères mais permanentes du pododerme peuvent entraîner des pododermatites aseptiques diffuses d'origine traumatique (GREENOUGH, 1982 ; Nilsson, 1982). Par ailleurs, cette affection est souvent associée à des troubles circulatoires au niveau des pieds, qui peuvent être favorisés par le manque d'exercice d'animaux confinés dans un espace restreint (GreENOUGH, 1982).

Des études complémentaires sont néanmoins nécessaires pour préciser dans quelle mesure un régime à base d'ensilage de maïs, pourrait provoquer un état d'acidose subclinique du rumen qui, associé à des conditions de logement difficiles, favoriserait la multiplication des cas de boiteries.

A ce propos, on peut interpréter de différentes manières la constatation que les animaux à fort potentiel de croissance boitent plus fréquemment que les autres ; dans une hypothèse d'étiologie alimentaire on peut estimer que ces animaux sont plus sensibles aux troubles métaboliques; dans une hypothèse d'étiologie traumatique on peut considérer que ces animaux, qui ont un poids vif plus élevé, sont aussi plus exposés.

Enfin, les relations mises en évidence entre croissance initiale, fréquence des boiteries et croissance totale montrent que des boiteries trop nombreuses dans les stations de testage sur la production de viande peuvent introduire des biais dans l'estimation de la valeur génétique des taureaux. Il s'agit en fait d'une interaction génotype-milieu, toujours difficile à prendre en compte dans l'indexation des reproducteurs.

\title{
Remerciements
}

A $\mathbf{M}^{\text {me }}$ et M. Guiller et à M. Regis de l'U.R.C.O. pour leur importante collaboration à ce travail.

\author{
Summary \\ Cases of lameness in fattening young bulls: clinical and organic findings, \\ effects on growth
}

Cases of lameness were systematically recorded for 14 months in young bulls from a progeny testing station in which growth was monitored monthly. The frequency of lameness was very high, with $2 / 3$ of the animals suffering from this condition, and 20 p. 100 severely. Clinical findings and photographs of the podal lesions showed that diffuse aseptic pododermatitis was responsible for most of these cases. A study of the livestock feeding conditions, failed to demonstrate that metabolic and nutritional etiological factors predominated over traumatic factors related to the housing of the animals on a hard, strawless floor with a $8 \mathrm{p}$. 100 slope on both sides of a central grate. The possible role and interaction of all these factors are discussed.

Statistical study of the relationship between lameness and growth rate by discriminatory analysis and multiple regression showed that : 1) lameness had a very highly significant effect on total daily mean gain : the weight increase during fattening decreased by $130 \mathrm{~g}$ per day in severe cases, 2) lame animals had a higher growth rate before lameness than 
unaffected animals. The significance of these results for understanding the etiology of diffuse aseptic pododermatitis, stock farm management, and the reliability of selection techniques, are discussed.

Key words : Lameness, young bull, growth, diffuse aseptic pododermatitis.

Reçu en mars 1983.

Accepté en septembre 1983.

\section{Références bibliographiques}

Bezille J., Boccara H., Paragon B.M., 1978. Les pieds des bovins, hygiène, soins, boiteries, 7-8, I.T.E.B., Paris.

Brent B.E., 1976. Relationship of acidosis to other feedlot aliments. J. Anim. Sci., 43, 930-935.

Cottereau P., 1967. La fourbure des bovins à l'engrais. Rev. Méd. Vét., 118, 827-837.

Greenough P.R., 1982. La fourbure - une revue. Quatrième symposium international sur les affections du pied des ruminants. Paris, 7-10 octobre 1982, 1-7, Société française de buiâtrie Maisons-Alfort.

Libersa M., 1981. Hiérarchie des problèmes pathologiques rencontrés chez les taurillons en Champagne. Bull. G.T.V., 2, 47-57.

Mahin L., Chaddli M., AdDi A., 1982. Observations sur les lésions et la fréquence de la pododermatite aseptique diffuse des bovins dans les conditions d'exploitations marocaines. Quatrième symposium international sur les affections du pied des ruminants, Paris, 7-10 octobre 1982, 1-4, Société française de buiâtrie Maisons-Alfort.

Mortensen K., Hesselhot M., 1982. La fourbure dans l'élevage laitier danois - une approche épidémiologique. Quatrième symposium international sur les affections du pied des ruminants. Paris, 7-10 octobre 1982, 1-7, Société française de buiâtrie Maisons-Alfort.

NiLsson S.A., 1982. La fourbure des bovins, étiologie et problèmes métaboliques. Quatrième symposium international sur les affections du pied des ruminants. Paris, 7-10 octobre 1982, 1-6, Société française de buiâtrie Maisons-Alfort. 